\title{
Understanding neurodynamical systems via Fuzzy Symbolic Dynamics
}

\author{
Krzysztof Dobosz ${ }^{\mathrm{a}}$, Włodzisław Duch ${ }^{\mathrm{b}, *}$ \\ ${ }^{a}$ Faculty of Mathematics and Computer Science, Nicolaus Copernicus University, Toruń, Poland \\ ${ }^{\mathrm{b}}$ Department of Informatics, Nicolaus Copernicus University, Toruń, Poland
}

\section{A R T I C L E I N F O}

\section{Article history:}

Received 11 February 2009

Received in revised form 23 November 2009

Accepted 10 December 2009

\section{Keywords:}

Symbolic dynamics

Neurodynamical system

Visualization of multidimensional time series

\begin{abstract}
A B S T R A C T
Neurodynamical systems are characterized by a large number of signal streams, measuring activity of individual neurons, local field potentials, aggregated electrical (EEG) or magnetic potentials (MEG), oxygen use (fMRI) or activity of simulated neurons. Various basis set decomposition techniques are used to analyze such signals, trying to discover components that carry meaningful information, but these techniques tell us little about the global activity of the whole system. A novel technique called Fuzzy Symbolic Dynamics (FSD) is introduced to help in understanding of the multidimensional dynamical system's behavior. It is based on a fuzzy partitioning of the signal space that defines a non-linear mapping of the system's trajectory to the low-dimensional space of membership function activations. This allows for visualization of the trajectory showing various aspects of observed signals that may be difficult to discover looking at individual components, or to notice otherwise. FSD mapping can be applied to raw signals, transformed signals (for example, ICA components), or to signals defined in the time-frequency domain. To illustrate the method two FSD visualizations are presented: a model system with artificial radial oscillatory sources, and the output layer (50 neurons) of Respiratory Rhythm Generator (RRG) composed of 300 spiking neurons.
\end{abstract}

(c) 2009 Elsevier Ltd. All rights reserved.

\section{Introduction}

Neuroimaging data and simulated neurodynamical systems are characterized by multiple streams of non-stationary data, and thus may be represented only in high-dimensional signal spaces. For example, functional magnetic resonance imaging (fMRI) provides thousands of streams corresponding to the changing activity of voxels, with sampling rate of a few hertz, and electroencephalographic (EEG) recordings hundreds of streams with sampling frequency of hundreds of hertz. High data volumes that quickly change in time make such signals very hard to understand. Popular signal processing techniques remove artifacts by various filtering techniques, involving waveform analysis, morphological analysis, decomposition of data streams into meaningful components using Fourier or Wavelet Transforms, Principal and Independent Component Analysis (PCA, ICA), etc. (Rangayyan, 2001; Sanei \& Chambers, 2008). Interesting events are then searched for using processed signal components, with time-frequency-intensity maps calculated for each component.

Such techniques are very useful, but do not show global properties of processes in the high-dimensional signal spaces. Simulation of complex dynamics is usually described in terms of at-

\footnotetext{
* Corresponding author.

E-mail address: wduch@is.umk.pl (W. Duch).
}

tractors, but precise characterization of their basins and possible transitions between them is rarely attempted. A mapping that separates interesting segments of the trajectory could help to categorize such events. Global analysis is needed to characterize different types of system's behavior, see how attractors trap dynamics, notice partial synchronization and desynchronization events, or filter the high frequency noise. For many applications (including brain-computer interfaces) a snapshot of the whole trajectory helping to understand its main characteristics, would be very useful. This is the goal of our paper, presenting a global approach to the high-dimensional signal analysis (to focus attention we shall talk about neurodynamics, although any dynamical system can be analyzed in this way).

Two inspirations have been important in the development of our approach. First, an observation that different brain areas probably "understand" and collaborate with each other by filtering the main properties of their large-scale activity, reacting to specific activations that may be roughly characterized in a symbolic way. The second inspiration comes from the successes of the symbolic dynamics (Hao \& Zheng, 1998) in understanding and simplifying the description of dynamical systems. Symbolic dynamics may be used as an approximation to brain processes if hard partitioning of the activity of various brain regions is done and labeled by a finite set of symbols. However, such a discretization may for most applications be either too rough or require too many symbols to be useful. The Fuzzy Symbolic Dynamics (FSD) introduced in 
this paper is based on a few membership functions rather than a large set of symbols. To see the trajectory $\{x(t)\}$ of the whole system, localized membership functions, or "probes" that are activated by the trajectories that pass near their center, are placed in the signal space. Using $k$ such membership functions $y_{i}(x(t))$, strategically placed in important points of the signal space, a nonlinear reduction of dimensionality suitable for visualization of trajectories is achieved. Inevitably a lot of details will be lost but with a proper choice of parameters the information that correlates with observed behavior or an experimental task may be preserved, while irrelevant information will be suppressed.

A long-term goal of this research is to find the brain-mind transformation that maps the trajectory representing measured neural activity to the psychological space with dimensions that represent perceptions, intentions and other inner events that are part of our mental life. Many important properties of neurodynamics should be reflected in such relatively lowdimensional psychological spaces (Duch \& Diercksen, 1995). The next section introduces the Fuzzy Symbolic Dynamics approach and defines the FSD mapping that captures some interesting properties of system's trajectories. To illustrate how to set up mapping parameters and how to interpret resulting images a very simple model of EEG sources is analyzed in Section 3, with radial and plain wave sources placed in a few points on a mesh, and sensors that record the amplitude of incoming waves in nodes of this mesh. As an example of real application in Section 4 visualization of trajectories of the neural Respiratory Rhythm Generator model (RRG) are analyzed. The final section contains a brief discussion with a list of many open questions.

The purpose of the visualization is to gain insight into general behavior of neurodynamical systems. For example, changing parameters of neurons will change the landscape of attractors that may potentially be reached. Although automatic quantization of some properties along the lines of recurrence plots, may be quite useful, this is beyond the topic of the present paper.

\section{Fuzzy Symbolic Dynamics}

Assume that some unknown sources create a multidimensional signal that is changing in time, for example an EEG signal measured by $n$ electrodes:

$x(t)=\left\{x_{i}(t)\right\} \quad i=1, \ldots, n t=0,1,2, \ldots$.

Vectors $x(t)$ represent the state of the dynamical system at time $t$, forming a trajectory in the signal space. Observing the system for a longer time should reveal the landscape created by this trajectory, areas of the signal space where the state of the system is found with the highest probability, and other areas where it never wonders. Recurrence maps (Marwan, Romano, Thiel, \& Kurths, 2007) and other techniques may be used to view some aspects of such trajectories, but do not capture many important properties that it reflects.

In the symbolic dynamics (Hao \& Zheng, 1998) the signal space is partitioned into regions that are labeled with different symbols, emitted every time the trajectory is found in one of these regions. The sequence of symbols gives a coarse-grained description of dynamics that can be analyzed using statistical tools. Dale and Spivey (2005) and Spivey (2007) argue that symbolic dynamics gives an appropriate framework for cognitive representations, although discretization of continuous dynamical states loses the fluid nature of cognition. Symbols obviously reduce the complexity of dynamical description because the partitioning of highly-dimensional signal spaces into regions with sharply defined boundaries is highly artificial. However, the symbolic approach may help to simplify the dynamics and make it more understandable. In fact the common practice of showing the differences that contrast two experimental conditions using averaged fMRI activations is an extremely simplified version of symbolic labeling that loses all dynamical information.

The notion of the symbolic dynamics is generalized in a natural way to a Fuzzy Symbolic Dynamics (FSD). Instead of discrete partitioning of the signal space leading to a set of symbols, interesting regions are determined analyzing probability density $p(x)$ of finding the trajectory $x(t)$ at some point $x$, averaging the results over time with an appropriate smoothing kernel, $p(x)=\sum_{i} K\left(x ; x\left(t_{i}\right)\right)$ (Duda \& Hart, 1973). Local maxima of this probability define quasi-stable states around which trajectories tend to cluster. Such maxima may serve as centers $\mu_{k}$ of prototypes associated with fuzzy membership functions $y_{k}\left(x ; \mu_{k}\right)$ that measure the degree to which the $x(t)$ state belongs to the prototype $\mu_{k}$. Membership functions may be defined using knowledge-based clustering (Pedrycz, 2005), or as prototypebased rules with context-based clustering techniques (Blachnik, Duch, \& Wieczorek, 2006). Context is defined by questions that are of interest, for example discrimination between different experimental conditions, or searching for invariants in one of these condition. Such methods will improve upon naive clusterization by automatically finding optimal parameters of membership functions that should reveal differences between various conditions.

For visualization Gaussian membership functions are quite useful:

$y_{k}\left(x ; \mu_{k}, \Sigma_{k}\right)=\exp \left(-\left(x-\mu_{k}\right)^{\mathrm{T}} \Sigma_{k}^{-1}\left(x-\mu_{k}\right)\right)$.

Diagonal dispersions $\Sigma_{k}$ are frequently sufficient, suppressing irrelevant signals, but in general covariance matrices (used in Mahalanobis distance) may extract more meaningful combinations of signals that correlate with experimental conditions, or with features of mapped signals that correspond to qualities of mental experience that are subjectively felt. Such a brain-mind mapping will be closer to the idea of cognitive representations than the symbolic dynamics that Dale and Spivey (2005) and Spivey (2007) advocate. They also stress "the continuity of mind", based on distributed patterns of neural activation. Such patterns may be approximated by fuzzy dynamics in a much better way than the purely symbolic description, generated by thresholding strongly activated prototypes. For example, sensorimotor actions cannot certainly be well approximated by symbolic labels.

Selecting only two or three prototypes is sufficient to visualize trajectories $x(t)$ in a two-dimensional space $y_{i}(t), y_{j}(t)$. For visualization each pair of functions should have sufficiently large dispersions $\sigma_{i}$ and $\sigma_{j}$ to cover the space between them, for example $\sigma_{i}=\sigma_{j}=\left\|\mu_{i}-\mu_{j}\right\| / 2$. Visualizations in three dimensions require plotting transformed points for three clusters, one for each dimension. Dispersions should then be set to the largest among the 3 pairs. Pairwise plots can be used to observe the trajectory from different points of view. Normalization of vectors in the signal space is assumed. To distinguish several experimental conditions optimization of parameters of membership functions should be done using context-based clustering techniques, creating clear differences in corresponding maps. Adding more localized functions in some area where dynamics is complex will show fine structure of the trajectory.

An alternative to fuzzy membership functions is to define reference points $R_{i}$ in the signal space, and measure the distance between the trajectory and these points $\left\|x(t)-R_{i}\right\|$, using some metric function. Non-linear metric functions should have some advantage in analysis of neurodynamics, as the influence of the trajectory on prototypes decreases sharply to zero with the distance, reflecting non-linear properties of neurons. We shall not consider here the problem of adaptation of membership function parameters, concentrating instead on the interpretation of global 

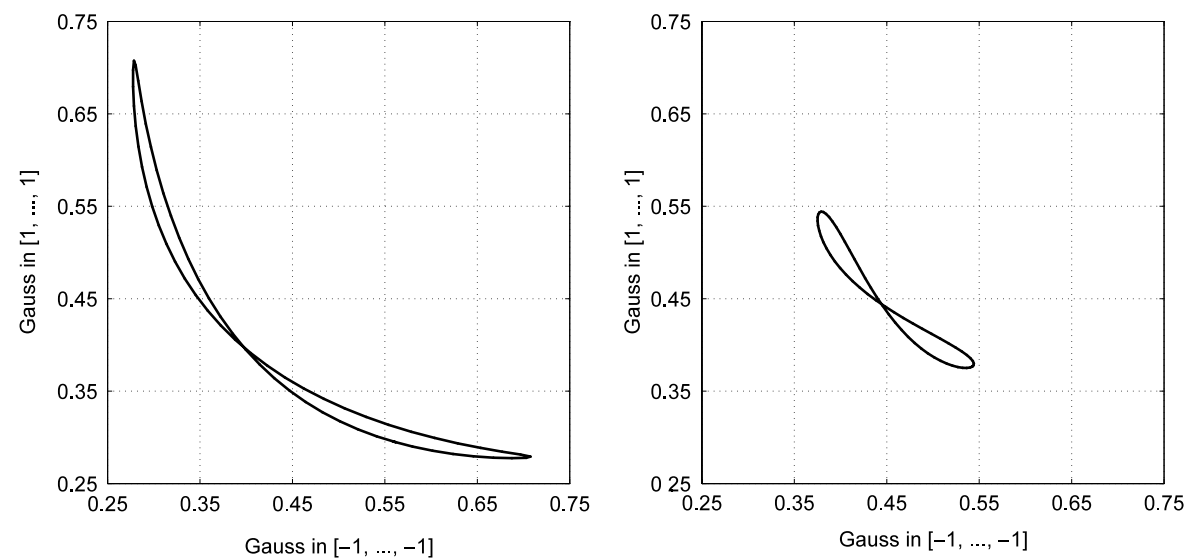

Fig. 1. Trajectories for one radial wave with the source at point $\left(\frac{1}{2}, \frac{1}{2}\right)$ (left side), and two radial waves with the sources at $\left(\frac{1}{4}, \frac{1}{4}\right)$ and $\left(\frac{3}{4}, \frac{3}{4}\right)$ (right side).

mappings. It is quite obvious that adaptation of these parameters will have crucial influence on the legibility of the mappings used for the visualization, and that trajectories that span the whole signal space may be localized if instead of a simple probe functions more complex density mixtures are used. Investigation of these issues is left for future research, here only a few examples showing the usefulness of the global trajectory analysis are reported. Dynamical systems with point attractors are obviously the easiest to analyze using FSD, membership functions may then be placed near the centers of these attractors estimated from the last part of the trajectory. We have analyzed neurodynamics of large layers in the Emergent simulator (O'Reilly \& Munakata, 2000) in this way, showing many details of the dynamics of such systems (Dobosz \& Duch, in preparation). Below two more difficult problems are presented.

\section{Plane and radial waves on a grid}

To see how various techniques may help to understand the structure of complex signals simple artificial models should be analyzed first. Sensors are placed on a quadratic grid with $n \times n$ points, where plane and radial waves generated by several sources are traveling, creating additive patterns and activating these sensors. Similar assumptions are made about electric potentials reflecting neuronal activity in the brain (for example, in the low resolution electromagnetic tomography, LORETA $\left.{ }^{1}\right)$.

The grid has equally spaced points $p_{i j}=\left(x_{i}, y_{j}\right)$ inside the square:

$x_{i}, y_{j} \in\left\{0, \frac{1}{n-1}, \ldots, \frac{n-2}{n-1}, 1\right\} \quad i, j=1, \ldots, n$.

The activation of the sensor due to a plane wave $F^{(l)}(t, x)$ traveling through the square in the grid point $p_{i j}$ at the time $t=0,1,2, \ldots$ is given by the equation:

$F^{(l)}\left(t, p_{i j}\right)=\cos \left(\omega_{l} t-\vec{k}_{l} \cdot \vec{p}_{i j}\right)$,

where $\omega_{l}$ is the frequency of the wave (defining time intervals), the wave vector $\vec{k}_{l}$ defines the direction of the wave movement and its length is equal to the inverse of the wave length and $\vec{p}_{i j}$ is the vector pointing to the grid point $p_{i j}$. Thus, for horizontal plane wave $\left(\vec{k}=\|\vec{k}\|[1,0]^{\mathrm{T}}\right)$ formula (4) becomes:

$F\left(t, p_{i j}\right)=\cos \left(\omega t-k x_{i}\right)$.

Radial wave reaching the sensor at grid point $p_{i j}$ leads to an activation:

1 See http://www.unizh.ch/keyinst/loreta.
$R^{(l)}\left(t, p_{i j}\right)=d\left(r^{(l)}\right) \cos \left(\omega_{l} t-k_{l} r^{(l)}\right)$,

where

$r^{(l)}=\sqrt{\left(x_{i}-x_{0}^{(l)}\right)^{2}+\left(y_{j}-y_{0}^{(l)}\right)^{2}}$

is the distance between point $p_{i j}$ and the wave source $\left(x_{0}, y_{0}\right)$, and $d\left(r^{(l)}\right)$ is the damping function, for example decreasing in a linear way from the sources.

The final activation $A\left(t, p_{i j}\right)$ of the sensor in point $p_{i j}$ at time $t=0,1,2, \ldots$ is obtained by summing and normalizing all wave amplitudes in every grid point:

$A\left(t, p_{i j}\right)=\left(\sum_{l=1}^{N_{f}} F^{(l)}\left(t, p_{i j}\right)+\sum_{l=1}^{N_{r}} R^{(l)}\left(t, p_{i j}\right)\right) /\left(N_{f}+N_{r}\right)$.

The $n \times n$ matrix $A(t)$ contains values of all sensors' activations at the time $t$. Elements of $A(t)$ are defined in the $n^{2}$-dimensional signal space and are normalized in the $[-1,+1]$ interval. Noise may be included by adding independent random numbers to each element.

Fuzzy membership functions (2) may serve as probes (detectors of activity) in this space. Placing their centers in two opposite vertices of the hypercube $s=[-1,1]^{n^{2}}$ :

$\mu_{1}=[-1, \ldots,-1]^{T} \quad \mu_{2}=[1, \ldots, 1]^{T}$

the membership functions take all $n^{2}$ sensor activations $A(t)$ as their argument:

$G_{k}\left(A(t) ; \mu_{k}, \sigma_{k}\right)=\exp \left(-\frac{\left\|A(t)-\mu_{k}\right\|}{2 \sigma_{k}^{2}}\right)$,

where $\sigma_{k}$ defines the dispersion.

A lot of experiments have been conducted using the $16 \times 16$ grid with 256 points (the maximum number of electrodes used in real EEG experiments), and various number of stationary and non-stationary sources, frequencies and directions. For this grid $\sigma_{1}=\sigma_{2}=\left\|\mu_{1}-\mu_{2}\right\| / 10$ gives relatively wide range of sensor activations. In Fig. 1 examples of trajectories for one and two radial waves are presented, using $\omega=0.1$, which is sufficient for smooth trajectory changes, and the wave vector length $\|k\|=2 \pi$. The specific position of sources and combinations of planar and radial waves may be identified with correct placement of centers and dispersions of the membership functions.

Static sources are not that much interesting. To see how a simple dynamics is mapped to FSD trajectories consider 3 radial sources, first placed at $(1 / 2,1)$ position and active from $[0,350]$ $\mathrm{ms}$, second at $(0,1 / 3)$ active from $[200,650]$, and third at $(2 / 3$, 

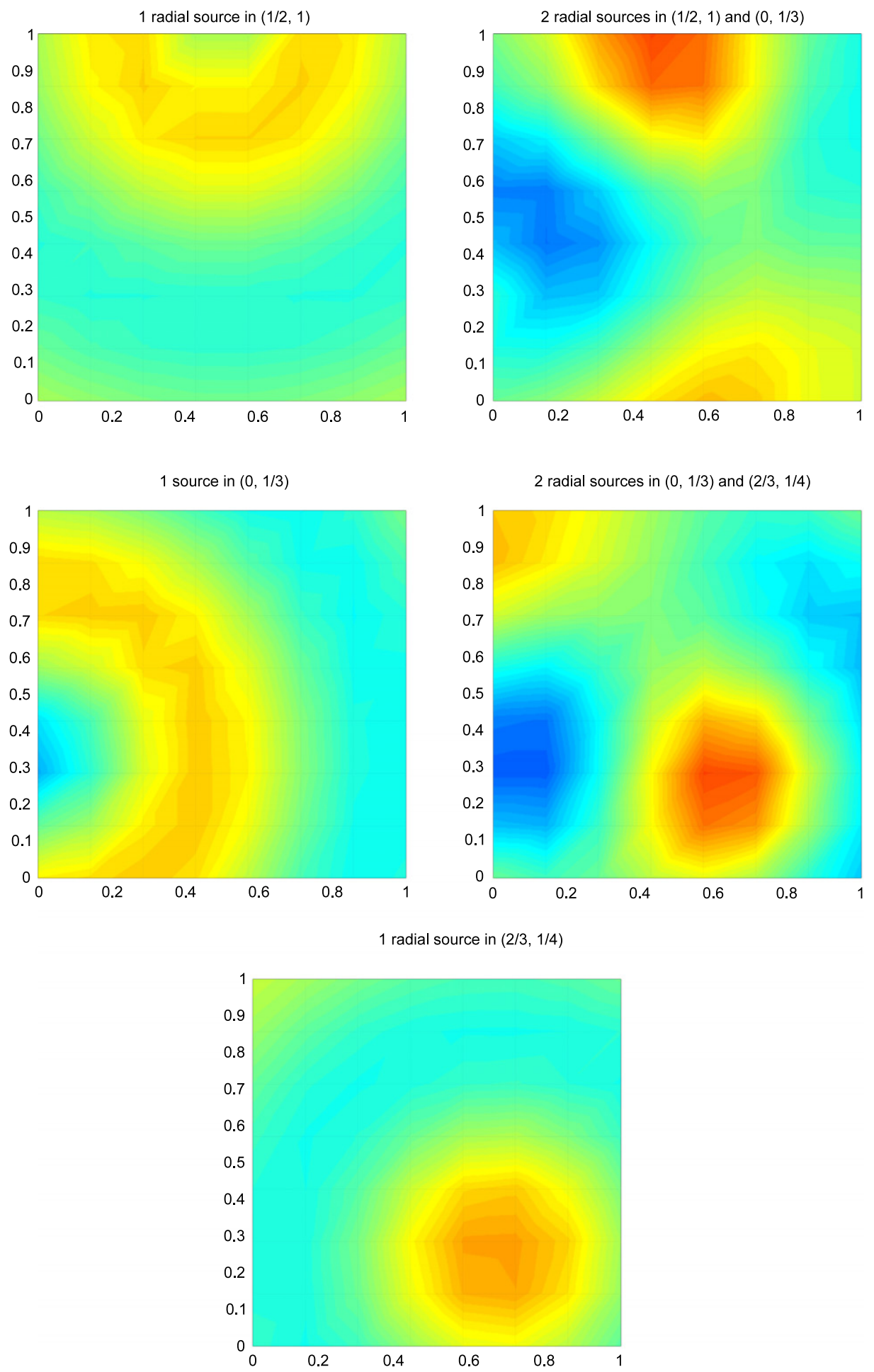

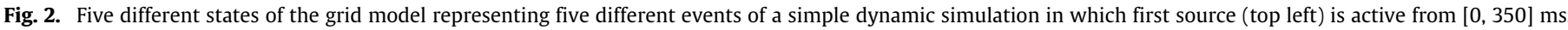

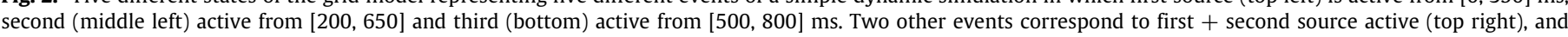
second + third source active (middle right).

1/4) active from $[500,800] \mathrm{ms}$. Thus there are 5 events: first source is active, first + second, second only, second + third, and third only. All of these events are shown in Fig. 2. Results of the FSD visualization are presented in Fig. 3 (bottom right). Although different attractors of this dynamics may be distinguished the sequence of events is not quite clear, and in the more complex case will be hard to unravel.

An interesting non-linear way to map trajectories into a low-dimensional space is to use kernel PCA (Schölkopf, Smola, \& Müller, 1998) that provides complex membership functions around probability density peaks, and thus helps to discriminate different events. Fig. 3 (top right) presents the kernel PCA plot of two components in which the first direction is close to the standard PCA (top left in Fig. 3), but the fourth direction nicely separates different segments of the trajectory. Components of PCA, kPCA and ICA were chosen in such a way that they best separate different attractors of the system trajectory. Choosing right components in such methods is a common problem in signal analysis and is usually done by careful inspection of components. The best components for visualization purposes were chosen by comparing 

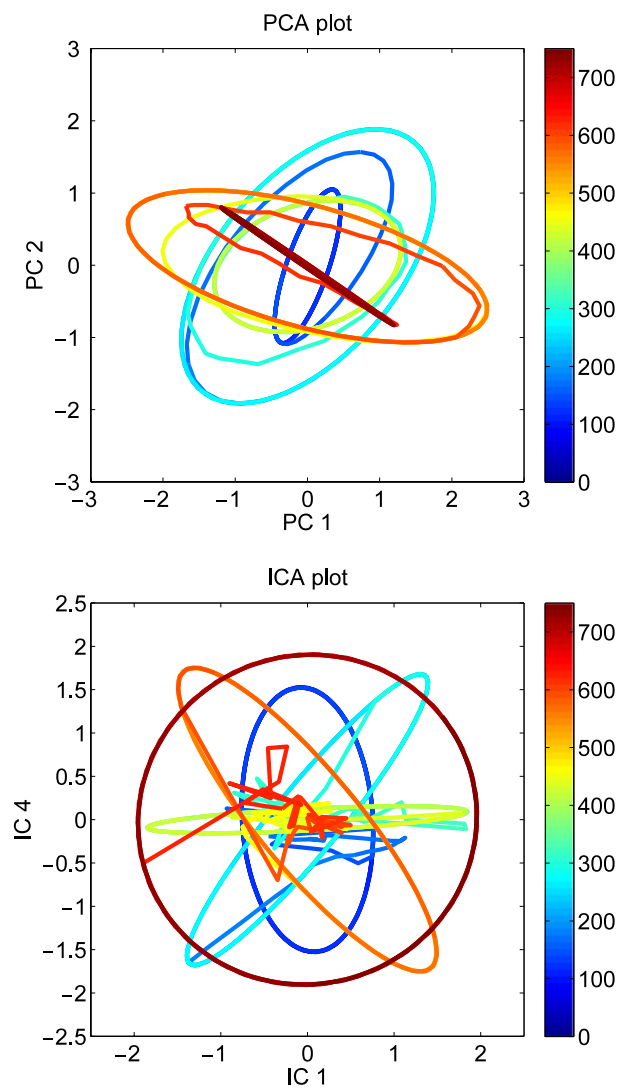
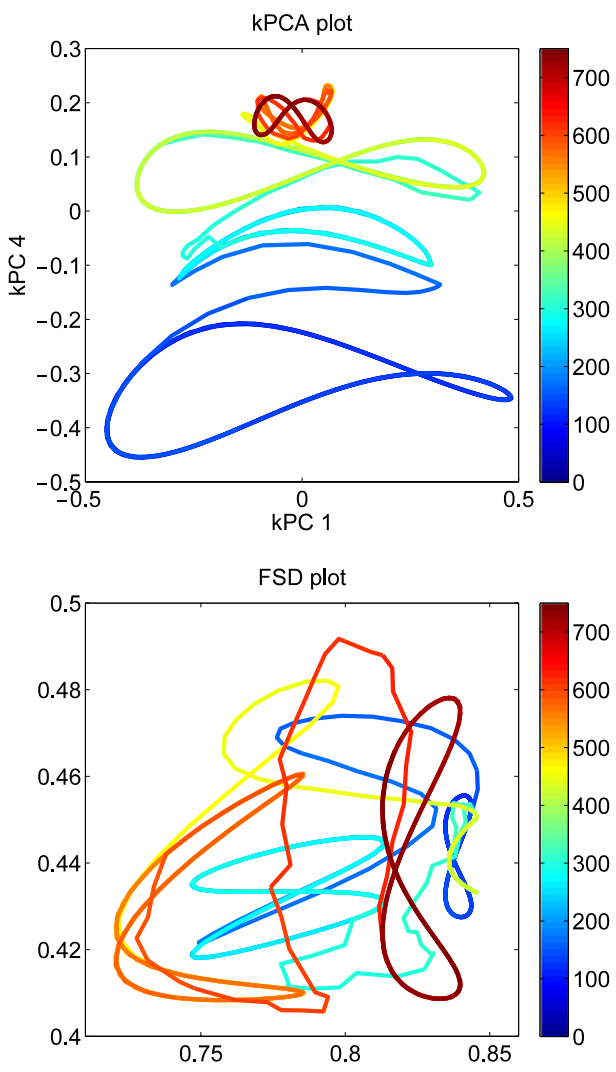

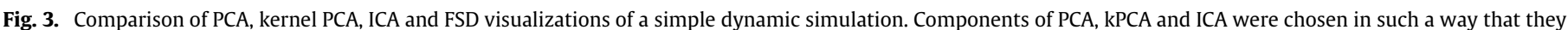

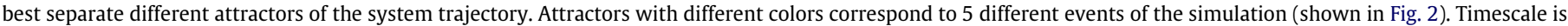
given by the bar on the right.
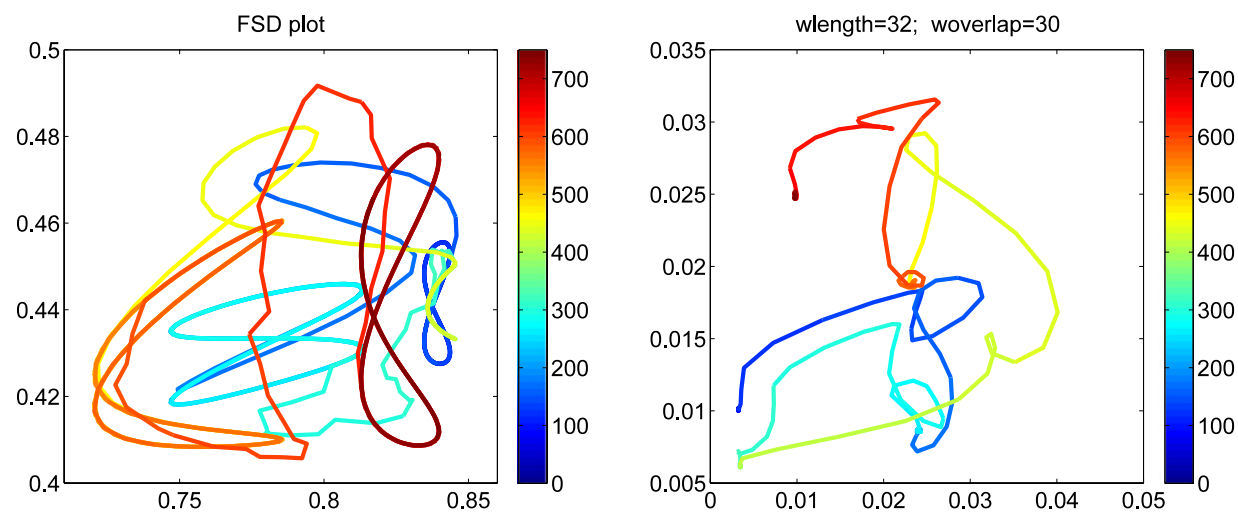

Fig. 4. FSD plot (left, the same as in Fig. 3), and an estimation of the standard deviation of original trajectory smoothed using Parzen windows (right).

plots for pairs of a few main components in each method; in PCA and KPCA components were in order of decreasing component variance; in ICA ordering was done by canonical correlation analysis. A satisfactory representation of the signal would be one in which different attractors are placed in different parts of the twodimensional space, but also the sequence of events is preserved. In the kernel PCA visualization the sequence is right, but still the last two attractors (corresponding to the last two events) overlap.

Additional smoothing may be applied in various ways, for example by moving from the original signals to their shorttime window Fourier transforms, followed by wavelet bump modeling (Vialatte et al., 2007). This will be especially important for analysis of real EEG data. Perhaps the simplest way to convert attractors from the FSD representation to more point-like sequences of different events is to consider standard deviation of the fuzzified signals in a time window that is long enough to cover full oscillation. Smoothing of these estimations is done using the Parzen window approach with rectangular kernel. The dimensionality is not changed but unique characteristics of each event are extracted, as shown in Fig. 4.

FSD is not an attempt to interpret only EEG data but a general approach to the visualization of trajectories of any highdimensional systems. Attempts to find clusters in the trajectories using Kohonen networks have been presented by Andras and Wennekers (2007), and such attempts may also be viewed as a step towards the Fuzzy Symbolic Dynamics. The activity of Kohonen network's nodes shows patterns of activity changing in time, not the whole trajectories, as in our approach.

\section{Visualization of the activity of respiratory rhythm generator}

FSD approach has also been used to study behavior of the neural Respiratory Rhythm Generator model (RRG). The RRG 


\section{ARTICLE IN PRESS}
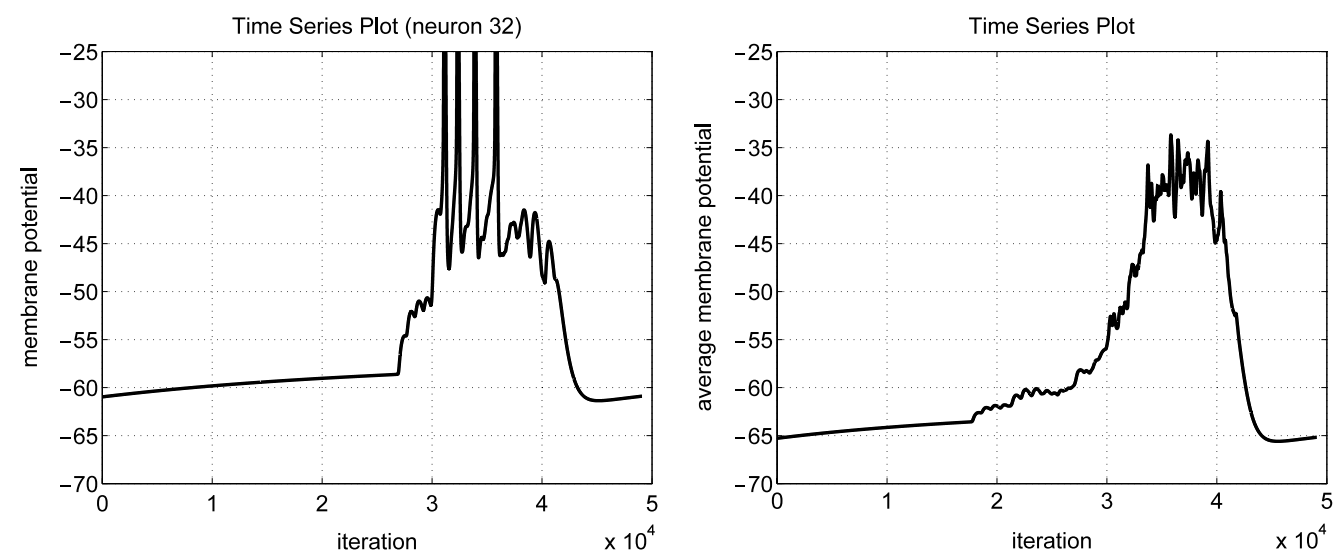

Fig. 5. The time series plot representing a membrane potential of a single neuron (left) and an average membrane potential of 50 neurons vs. iteration number (right).
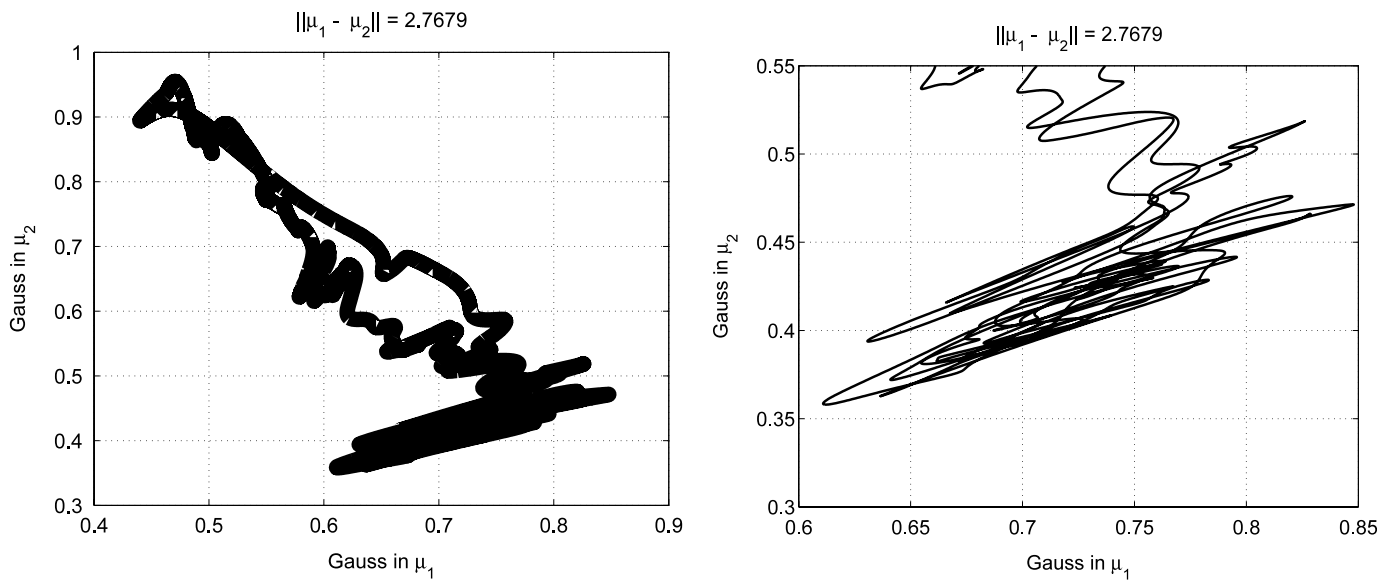

Fig. 6. The FSD map of the trajectory of 50 neurons (left), and zoomed area of the trajectory for the time series with one burst around the main attractor.
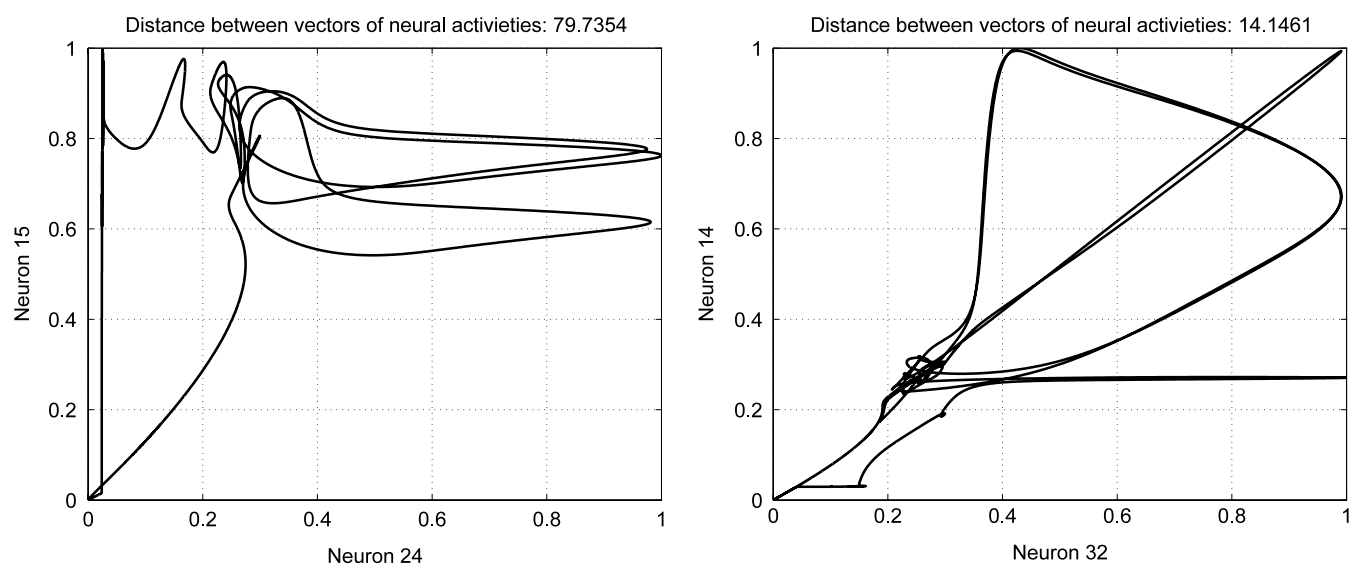

Fig. 7. Neural activity plots for 2 neurons from RRG that have the most (left) and the least (right) different vectors of neural activities.

is a parametric neural network model constructed from three populations of spiking neurons: beaters (200 in the model), bursters (50 units) and followers (50 units). The last population provides an output of the model that is used for synaptic excitation of motoneurons and in consequence controls upper and lower lung muscles. Our implementation of RRG is based on the spiking neural network model proposed by Butera, Rinzel, and Smith (1999).

Below visualization of the followers (output layer neurons) is examined. The membrane potential of a single neuron and the average membrane potential of all 50 output neurons is shown in Fig. 5. The first trajectory for time series corresponding to a single burst is presented in Fig. 6 (left). The number of samples along these trajectory was equal to 49090 , each vector containing membrane potentials of all 50 follower cells (normalized in every dimension). Clusterization was done with the $k$-means algorithm, for two clusters where Gaussian probe functions have been placed. Trajectories have been drawn with a thick pen to account for a jitter that blurs them when longer time sequences are taken.

Fig. 6 (right) shows trajectory for the same time series as Fig. 6 (left), zooming on one of the attractors to show details of oscillations around it. This attractor corresponds to oscillations visible in the highest part of the time series plot (Fig. 5). Generating more bursts slowly fills the whole area with trajectories giving almost uniform probability of finding the system there. This shows restricted chaotic behavior of the system at the peak of activity. 

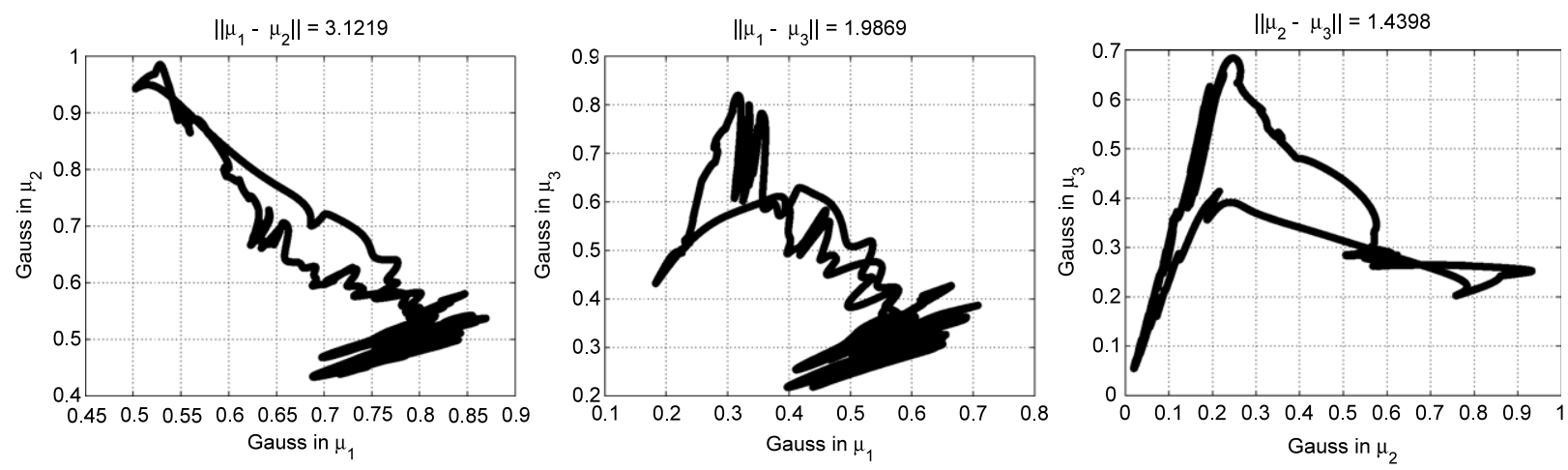

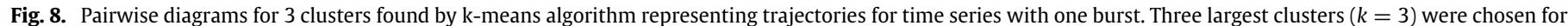

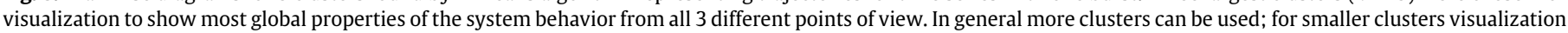
will show local properties of the system.
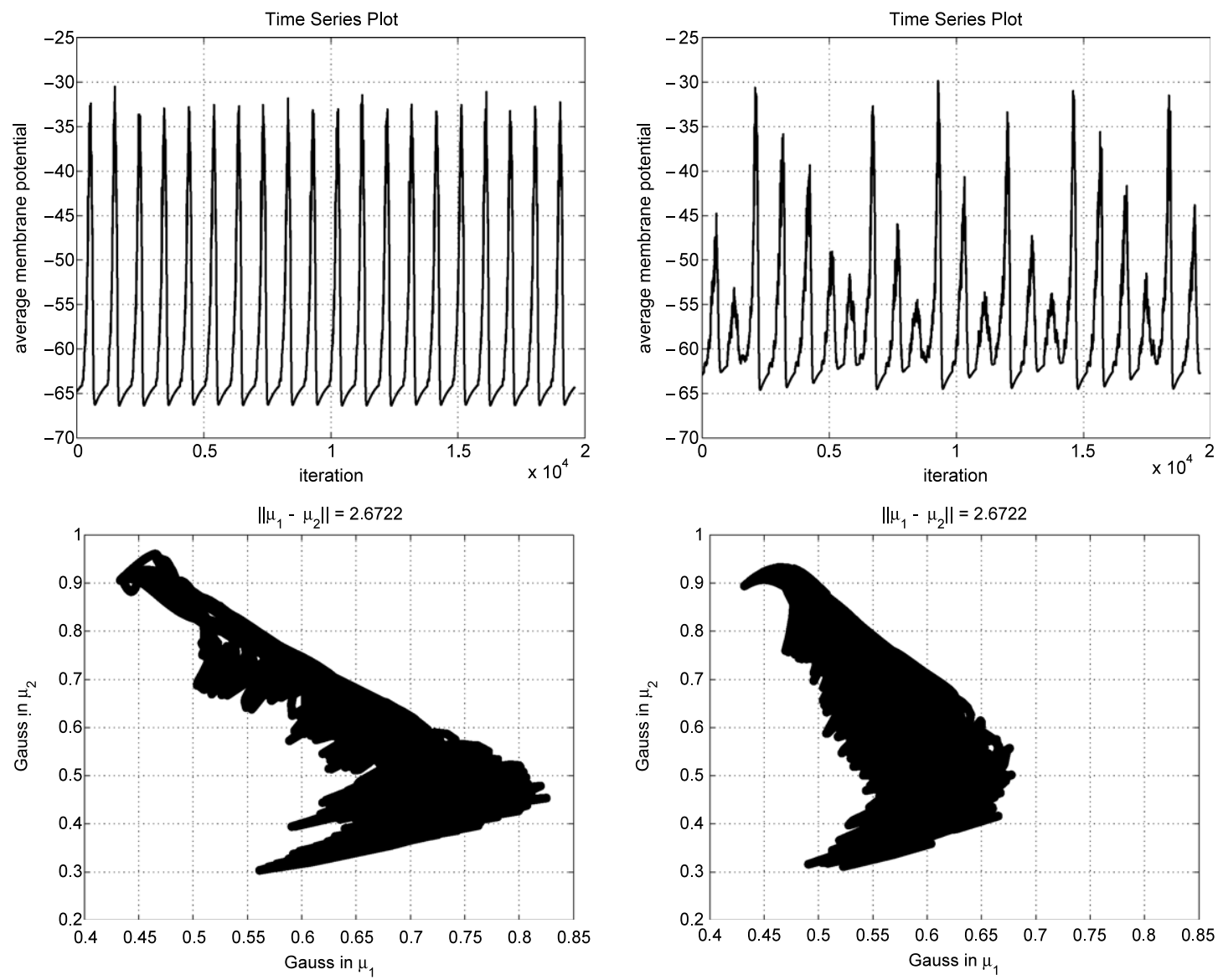

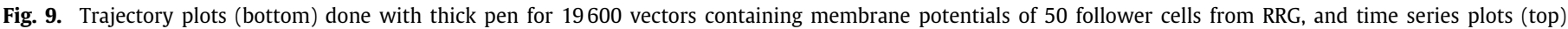

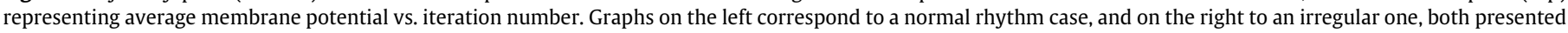
using the same membership functions.

A common visualization technique in the analysis of neural dynamics is to show plots of activations for selected pairs of neurons. In Fig. 7 two different pairs are shown. Unfortunately with 50 neurons there are 1225 possible pairs and most of them show quite different plots. Thus the pairwise visualization of single neuron activity does not provide much useful information. The global dynamics is much more stable.

In the FSD approach three cluster centers are defined using the $k$-means algorithm $(k=3)$. Pairwise diagrams show trajectories for all three cluster pairs (Fig. 8). Distances between cluster centers are printed above the graphs. The second pair is more sensitive to variability that appears during building of the discharge activity, showing quite a bit of variance in this process.
The RRG model may generate various rhythms that correspond to different breathing patterns. Trajectory examples in Fig. 9 compare two distinct cases, one for normal, regular burst generation, and one for irregular case with different burst strengths (i.e. different peak heights). The trajectories have been drawn using 19600 vectors, each containing values of membrane potentials of 50 follower cells, covering about 20 spikes. Two clusters have been found using the $k$-means algorithm, and the same parameters of membership functions used in both cases. Irregular case seems to reach the same amplitude but as a whole behaves quite differently, reaching much smaller values in the first dimension, due to the lack of synchronization between different output neurons. 

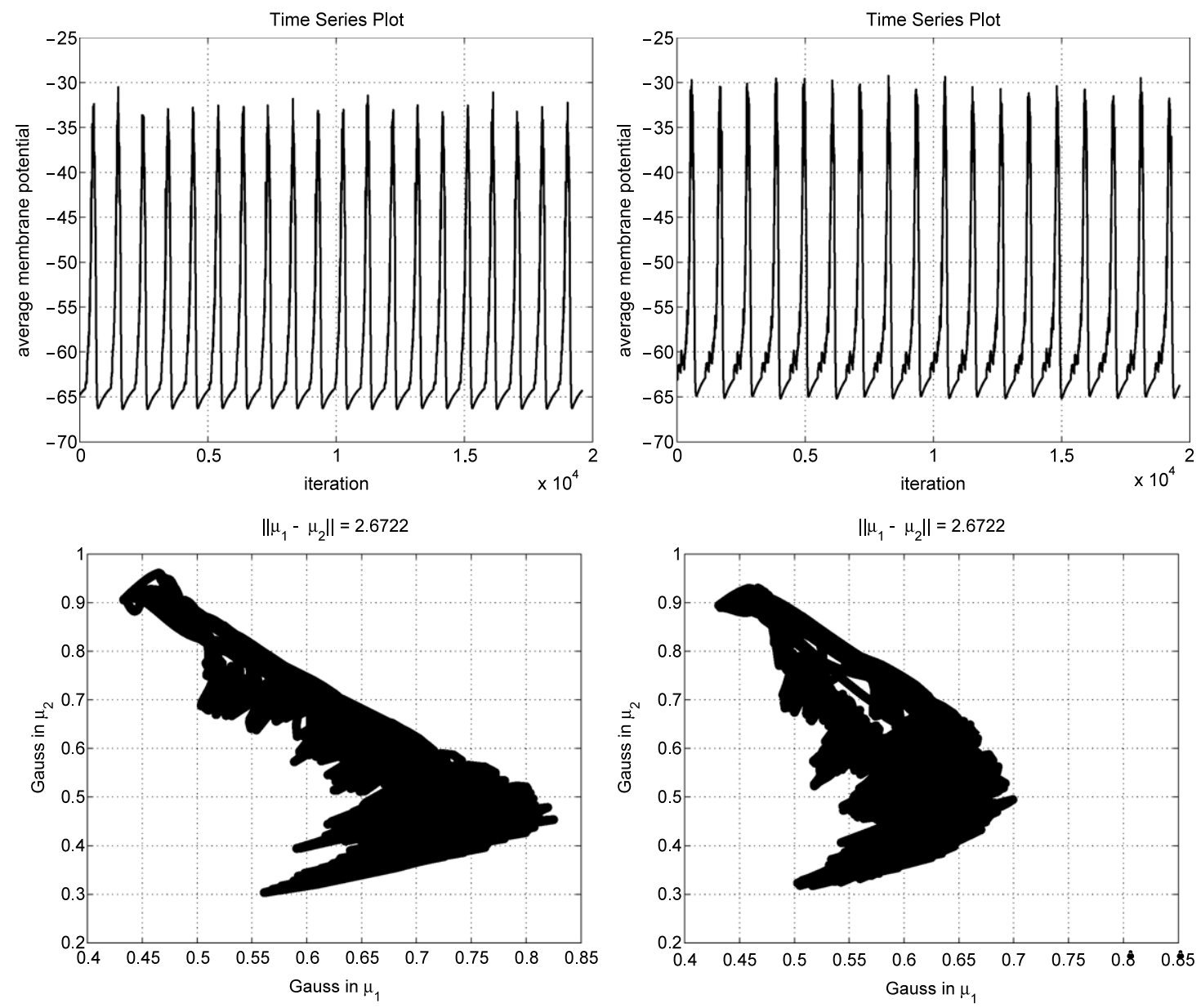

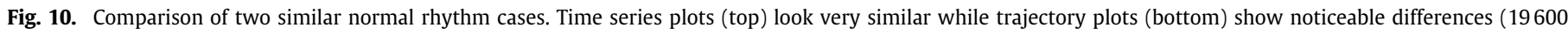
points).

When two similar time series plots are compared small differences between them may not be noticeable. The FSD method is sensitive to small changes in the global dynamical state and consequently it allows for a quite accurate comparison. Fig. 10 compares two normal rhythms that differ only slightly. Time series plots looks very similar but the global trajectories in the FSD graphs show significant differences.

In all examples presented in this section dispersions of Gaussians were set to the half of the distance between centers $\left(\left\|\mu_{1}-\mu_{2}\right\| / 2\right)$. The complexity of the FSD map generation depends on a procedure that is used to find parameters of membership functions (centers and dispersions), generation of the map requires only $O(n)$ operations for a $n$-point trajectory.

The last example of the FSD mapping presents the visualization of a large semantic layer in the model of dyslexia implemented in the Emergent simulator (see O'Reilly \& Munakata, 2000, chap. 10). This model has full bidirectional connectivity between orthography $(6 \times 8$ units $)$, phonology $(14 \times 14)$, and semantic layers $(10 \times 14)$, with recurrent self-connections within each of these layers, and additional hidden layers of neurons between each of these 3 layers. The model has been trained on 40 words, half of them concrete and half abstract. Semantics has been captured by using 67 features for concrete words (with average of 18 active features per word) and 31 for the abstract ones (about 5 active features on average), with half of the semantic layer devoted to abstract and half to concrete features. The correlation dendrogram between all 40 words is presented in (O'Reilly \& Munakata, 2000 , chap. 10 , fig. 10.7). The model was slightly modified by introducing the accommodation mechanism into neuronal units that allows the system to evolve unhampered. Also some synaptic Gaussian noise (with zero mean and 0.02 variance) was present to provide an extra energy for the system.

In Fig. 11 trajectories and attractor basins of 4 pairs of correlated words are displayed, with concrete words in the first two pairs (flag, coat, hind, deer) and abstract words (wage, coat, loss, gain) as the last two pairs. The system has quite complex dynamics but the FSD visualization shows how the system is evolving over time. Trajectories progressively pass between different basins of attraction which are represented by dense spots of trajectory points. Some attractors may be difficult to reach which is indicated by chaotic trajectories that lead to them. Both plots present evolution of the model with the same set of parameters. Substantial differences between certain trajectories of corresponding words are a consequence of noise randomness and significant influence of the accommodation mechanism on the model behavior. For visualization purpose three Gaussian membership functions were used with centers randomly drawn from the uniform distribution over $[0,1]$ and dispersions set to value 2.0. For each word evolution of the system last precisely 500 cycles (roughly 1 cycle correspond to 5-10 ms of simulated real time).

\section{Discussion}

The symbolic dynamics has found many applications (Dale \& Spivey, 2005; Hao \& Zheng, 1998), while its fuzzy version, to the best of our knowledge, has never been developed. The Fuzzy Symbolic Dynamics is based on the fuzzy partitioning of the state space 
Activation in Semantics layer [dyslex.proj]

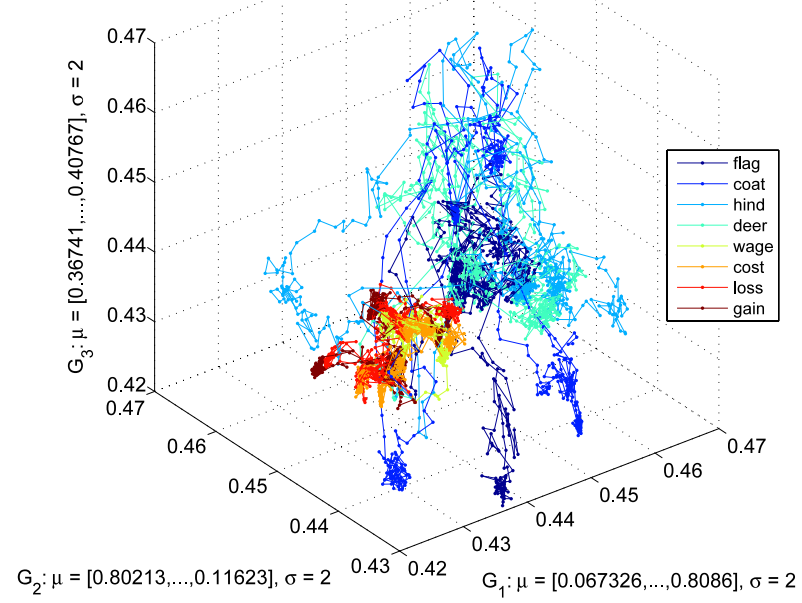

Activation in Semantics layer [dyslex.proj]

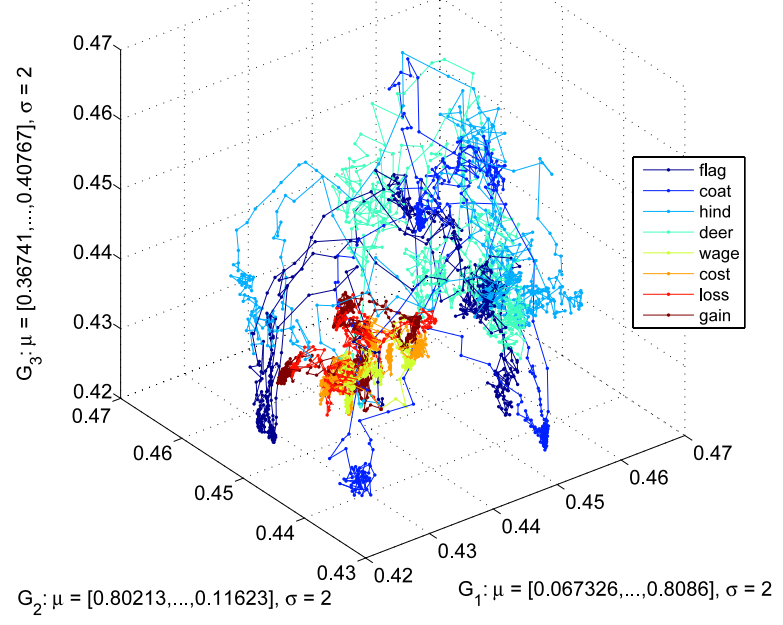

Fig. 11. Comparison of two different trials of the dyslexia model with the same set of parameters. Substantial differences between certain trajectories of corresponding words are a consequence of noise randomness and significant influence of the accommodation mechanism on the model behavior.

and thus is a generalization of the discrete classical symbolic dynamics (Cvitanović, Artuso, Mainieri, Tanner, \& Vattay, 2008) that may be obtained from the fuzzy version by thresholding the activations of membership functions. Instead of transition matrices estimating probability of transitions between the symbols more precise information about the global trajectories is retained. Recurrence plots are another way to understand some aspects of global trajectories of high-dimensional dynamical systems by visualization (Marwan et al., 2007). The FSD should thus find as many applications as the classical symbolic dynamics and recurrence plots. In particular cognitive science has reached the point where moving from finite automata models of behavior to continuous dynamical models of cognition becomes essential (Spivey, 2007).

In this paper we have focused only on FSD visualizations, showing that interesting events and irregular states may sometimes be easier to discover using global plots rather than looking at specific components of signals. Many aspects of dynamics may be analyzed using this technique:

1. In which part of the signal space the state of the system spends most of its time?
2. How many attractors can be identified?

3. What are the properties of attractor basins (size, depths, time spend)?

4. What are the probabilities of transition between them?

5. How fast transitions occur?

6. What type of oscillations occur around attractors?

FSD visualizations lose many details that are contained in the original trajectories, but allow to focus on specific areas near interesting events and analyze them more precisely using membership functions that are adapted to local probability density distributions. The trick is to retain interesting information, suppressing the chaotic, random part. The choice of membership functions in FSD gives sufficient flexibility to achieve this. Many quantitative measures allowing to compare different dynamical systems may be introduced on FSD mappings, for example:

- the number of attractors;

- percentage of time spent by the system in a given attractor basin;

- character of oscillations around attractors, including some measures of chaos;

- distances between attractors, measured by the time of transitions;

- probabilities of system transitions between attractors.

Such measures allow for interesting characterization of dynamical systems. Application of the FSD to recurrent networks shows transitions between attractor states. To see properties of attractors noise $\epsilon$ of increasing variance may be introduced to explore their basins, and local variance of the trajectories $\operatorname{var} x(t, \epsilon)$ investigated as its function. Properties of point attractors in simulated systems are relatively easy to analyze by plotting the relation between noise level and the variance of the trajectory near the attractor. For quasiperiodic attractors the variance in the direction perpendicular to the trajectory may be estimated. In some experimental situations the level of noise in the system may also be partially controlled.

Applications to real EEG signals will require careful optimization of membership functions, with conditional clustering to remove irrelevant information by finding most informative center locations and weights for different signals. One approach that we are exploring here is based on smoothing the EEG signals through bump modeling (Vialatte et al., 2007), finding combinations of signals that are important for discrimination of mental events - this is done through optimization of the projection pursuit based on Quality of Projected Clusters (Grochowski \& Duch, 2008) - and applying the FSD to visual multidimensional signals after all transformations. Direct FSD visualization of the whole EEG signal does not show any interesting properties because of the high complexity of EEG data and many irrelevant components, therefore without initial preprocessing the FSD approach (as well as any other visualization techniques) will not extract useful structures from the signal.

The visualization of highly-dimensional trajectories obviously depends on what aspects of the system behavior is of interest. Methods of parameter adaptation that include context (Blachnik et al., 2006; Pedrycz, 2005) will soon be applied to the visualization of real experimental data. For strongly non-stationary signals the whole landscape containing basins of attractors may slowly rotate, preserving relations between main attractors. For example, change in the level of neuromodulation may influence the landscape by increasing the overall activations in some regions of signal space. Parametrization of probes that should then change in time to counter this effect would be important. The great challenge is to find quantitative measures of the FSD representations that would be useful in brain-computer interfaces, and to find meaningful combinations of signals correlated with mental events and inner experiences. 


\section{References}

Andras, P., \& Wennekers, T. (2007). Cortical activity pattern computation. BioSystems, 87, 179-185.

Blachnik, M., Duch, W., \& Wieczorek, T. (2006). Selection of prototypes rules: Context searching via clustering. In Lect. notes artif. intell.: vol. 4029 (pp. 573582 ).

Butera, R., Rinzel, J., \& Smith, J. (1999). Models of respiratory rhythm generation in the pre-Bötzinger complex: I. Bursting pacemaker neurons; II. Populations of coupled pacemakers. Journal of Neurophysiology, 82, 382-397; 398-415.

Cvitanović, P., Artuso, R., Mainieri, R., Tanner, G., \& Vattay, G. (2008). Chaos: Classical and quantum. Copenhagen, Denmark: Niels Bohr Institute, URL http:// ChaosBook.org.

Dale, R., \& Spivey, M. J. (2005). From apples and oranges to symbolic dynamics: A framework for conciliating notions of cognitive representation. Journal of Experimental and Theoretical Artificial Intelligence, 17(4), 317-342.

Duch, W., \& Diercksen, G. H. F. (1995). Feature Space Mapping as a universal adaptive system. Computer Physics Communications, 87, 341-371.

Duda, R., \& Hart, P. (1973). Pattern classification and scene analysis. John Wiley \& Sons.
Grochowski, M., \& Duch, W. (2008). Projection pursuit constructive neural networks based on quality of projected clusters. In Lect. notes comput. sci.: vol. 5164 (pp. 754-762).

Hao, B., \& Zheng, W. (Eds.). (1998). Applied symbolic dynamics and chaos. Singapore: World Scientific.

Marwan, N., Romano, M. C., Thiel, M., \& Kurths, J. (2007). Recurrence plots for the analysis of complex systems. Physics Reports, 438, 237-329.

O'Reilly, R. C., \& Munakata, Y. (2000). Computational explorations in cognitive neuroscience. Cambridge, MA: MIT-Press.

Pedrycz, W. (2005). Knowledge-based clustering: From data to information granules. Chichester, England: Wiley-Interscience.

Rangayyan, R. M. (2001). Biomedical signal analysis: A case-study approach. New York: Wiley-IEEE Press.

Sanei, S., \& Chambers, J. A. (2008). EEG signal processing. Chichester, England: Wiley.

Schölkopf, B., Smola, A. J., \& Müller, K.-R. (1998). Nonlinear component analysis as a kernel eigenvalue problem. Neural Computation, 10, 1299-1319.

Spivey, M. J. (2007). The continuity of mind. New York: Oxford University Press.

Vialatte, F. B., Martin, C., Dubois, R., Haddad, J., Quenet, B., Gervais, R., et al. (2007) A machine learning approach to the analysis of time-frequency maps, and its application to neural dynamics. Neural Network, 20(2), 194-209. 